\title{
An Entropy based Video Watermarking Scheme
}

\author{
Angshumi Sarma \\ Dept. of Electronics \& Communication Engineering \\ IST, Gauhati University \\ Guwahati, Assam, India
}

\author{
Amrita Ganguly \\ Dept. of Electrical Engineering \\ Assam Engineering College \\ Guwahati, Assam, India
}

\begin{abstract}
The emerging interest in the field of digital watermarking is due to the increase in concern over copyright protection of digital information and prevention of unauthorized access, duplication and manipulation of digital data. In this paper, an entropy based blind video watermarking scheme is proposed using combined DCT-DWT transform. Two level DWT is performed on the extracted video frames, which results in sixteen subbands. Entropy is calculated for all the sixteen subbands and the subband with the highest entropy is chosen for embedding the watermark bits. 8x8 block based DCT is performed on this subband and the middle frequency transformed coefficients of each block are selected for embedding the watermark bits. The watermarked video frames are QPSK modulated and transmitted through an AWGN channel. At the receiver end the watermarked frames are QPSK demodulated and the watermark is extracted. This scheme applied to videos shows that it is highly imperceptible and exhibits high robustness against additive white Gaussian noise.
\end{abstract}

\section{General Terms}

Security.

\section{Keywords}

Digital Video Watermarking, Entropy, Discrete Wavelet Transform (DWT), Discrete Cosine Transform (DCT), Robustness, Imperceptibility.

\section{INTRODUCTION}

In recent years, with the rapid development in the field of information technology and multimedia, digital media has become the primary means of communication in almost all aspects of our life along with the wide use of the network. At the same time, digital information security problem has emerged as a threat. Digital data can easily be accessed and manipulated without error, putting the rights of their owners at risk. Hence, copyright protection has become an urgent requirement. Digital watermarking has emerged as a solution to this problem. Digital watermarking can be defined as the process of embedding a certain piece of information (known as watermark) into multimedia content including text documents, images, audio or video streams, such that the watermark could be detected or extracted later in order to provide copyright protection to the data and prevent illegal copying.

Any digital watermarking scheme should possess two qualities- Robustness and Visual Imperceptibility. Robustness is a measure of immunity of the watermark against different modification and manipulation attempts. It is measured in terms of a correlation factor, calculated between the original watermark and extracted watermark. Its value is generally 0 to 1. Ideally it should be 1 but the value 0.7 is acceptable [1]. Imperceptibility is related to the quality of host object in presence of the watermark. It is measured by the PSNR (Peak signal to Noise Ratio). High PSNR indicates better imperceptibility of the watermarked video.

Many digital watermarking schemes have been proposed in literature for still images and videos [2, 3, 4, 5]. Current digital watermarking techniques may be grouped into spatialdomain techniques and frequency-domain techniques. Compared to spatial domain watermarking techniques, frequency-domain watermarking techniques proved to be more effective in achieving the imperceptibility and robustness requirements of digital watermarking algorithms $[6,7]$. Commonly used frequency-domain transforms are the Discrete Wavelet Transform (DWT), the Discrete Cosine Transform (DCT) and Discrete Fourier Transform (DFT). However, DWT has been used in digital image and video watermarking more frequently due to its time/frequency decomposition characteristics and its resemblance to the theoretical models of the human visual system [7]. DWTbased watermarking algorithms could further be improved by combining DWT with DCT $[6,7]$. The combination of the two transforms namely the DWT and the DCT could compensate for the drawbacks of each other, thus resulting in effective watermarking.

In this paper, an entropy based video watermarking scheme is proposed using DCT and DWT. Frames are extracted from the video and after necessary preprocessing, two level DWT is performed on each frame which results in sixteen subbands. Entropy is calculated for the sixteen subbands and the subband with the highest entropy is chosen for embedding the watermark bits. This subband is divided into $8 \times 8$ non overlapping blocks and DCT is carried out on them and the watermark bits are embedded into the sixteen middle frequency DCT coefficients. Two secret keys are used in this scheme. The first key contains the highest entropy subband numbers for all the video frames and the second key is used for generating the PN sequences for watermark embedding and extraction.

\section{THEORETICAL BACKGROUND}

The proposed work requires certain theoretical considerations related to Entropy, Discrete Cosine Transform (DCT) and Discrete Wavelet Transform (DWT). The following sections contain a brief description of these concepts. 


\subsection{Entropy}

Entropy is one of the statistical means to measure the texture content of an image or a video frame. Texture of an image provides measures of properties such as smoothness, coarseness and regularity. Human eye is generally insensitive to high entropy areas. Hence, if watermark is embedded in high entropy areas of an image or video frame then higher imperceptibility can be obtained.

\subsection{Discrete Cosine Transform (DCT)}

The discrete cosine transform (DCT) is a block based transform. It represents an image as a sum of sinusoids of varying magnitudes and frequencies [1, 7]. For an input image, $\mathrm{X}$, of size $\mathrm{N} \times \mathrm{N}$ the DCT coefficients for the transformed output image, Y, are computed according to (1). $X(i, j)$ is the intensity of the pixel in row $i$ and column $j$ of the image, and $\mathrm{Y}(\mathrm{u}, \mathrm{v})$ is the DCT coefficient in row $\mathrm{u}$ and column $\mathrm{v}$ of the DCT matrix.

$\mathrm{Y}(\mathrm{u}, \mathrm{v})=\mathrm{Cu}_{\mathrm{u}} \sum_{i=0}^{N-1} \sum_{j=0}^{N-1} \cos \frac{((2 j+1) y \pi)}{2 N} \cos \frac{((2 i+1) x \pi)}{2 N}$

where

$$
\begin{aligned}
& \mathrm{Cu}_{\mathrm{u}}, \mathrm{C}_{\mathrm{v}}=\sqrt{\frac{1}{N}} \text { for } \mathrm{u}, \mathrm{v}=0 \text { and } \\
& \mathrm{Cu}_{\mathrm{u}}, \mathrm{C}_{\mathrm{v}}=\sqrt{\frac{2}{N}} \text { for } \mathrm{u}, \mathrm{v}=1,2, \ldots \ldots \mathrm{N}-1
\end{aligned}
$$

Performing DCT of an image or video frame gives rise to three different frequency coefficient sets: low frequency, mid frequency and high frequency coefficient sets $[7,8]$. The DCT has a special property that most of the visually significant information of the image is concentrated in just a few coefficients of the DCT [1]. This is referred to as the 'Energy compaction Property'. Most of the signal energy is concentrated at the low frequency coefficients, which contains the most important visual parts of the image. And high frequency coefficients of the image are usually removed through compression and are more prone to noise attacks. Hence, watermark is embedded in the mid frequency coefficients so that the visibility of the image is not affected and the watermark is not removed by compression or noise attacks.

\subsection{Discrete Wavelet Transform (DWT)}

Wavelet transform decomposes an image or video frame into a set of band limited components which can be reassembled to reconstruct the original image without error [7, 9]. For 2-D images, applying DWT corresponds to processing the image by 2-D filters in each dimension. The filters divide the input image into four non-overlapping multi-resolution sub bands, a lower resolution approximation image (LL1), horizontal (HL1), vertical (LH1) and diagonal (HH1) detail components. The process can be repeated to obtain multiple scale wavelet decomposition. One of the advantages of DWT over DCT is that it can more accurately model the aspects of the HVS as

\begin{tabular}{|c|c|c|c|c|c|c|}
\hline \multirow{2}{*}{$\omega_{1}$} & \multicolumn{2}{|c|}{ HL1 } & 1 & 2 & 5 & 6 \\
\hline & & & 3 & 4 & 7 & 8 \\
\hline \multirow{2}{*}{ LH1 } & แ2 & HL2 & 9 & 10 & 13 & 14 \\
\hline & LH2 & HH2 & 11 & 12 & 15 & 16 \\
\hline
\end{tabular}
compared to DCT $[3,5]$.
Fig 1: Two level Wavelet Decomposition and subband numbering

\subsection{Performance Measures}

The performance of any watermarking scheme can be evaluated on the basis of its robustness and imperceptibility. The imperceptibility of the scheme is measured by a quantitative measure called the Peak Signal to Noise Ratio (PSNR), calculated between the original video frame and the corresponding watermarked frame. The PSNR $[1,2,6,10,11]$ is given by

$$
\text { PSNR }=10 \log \frac{(255)^{2}}{M S E} d B
$$

Where MSE is the Mean Square Error given by

$$
\mathrm{MSE}=\frac{1}{M * N} \sum_{i=1}^{M} \sum_{j=1}^{N}\left(X_{i j}-W_{i j}\right)^{2}
$$

Where $\mathrm{X}_{\mathrm{ij}}$ is a pixel of the Original frame and $\mathrm{W}_{\mathrm{ij}}$ is a pixel of the Watermarked frame of size $\mathrm{MxN}$.

The robustness of any scheme can be measured by a quantitative measure called the Normalized Correlation (NC), calculated between the original watermark and the recovered watermark. The Normalized Correlation $[1,2,6,12]$ is given by

$$
\mathrm{NC}=\frac{\sum_{i=1}^{M} \sum_{j=1}^{N} W_{o i j} * W_{r i j}}{\sqrt{\sum_{i=1}^{M} \sum_{j=1}^{N} W_{o i j}^{2}} \sqrt{\sum_{i=1}^{M} \sum_{j=1}^{N} W_{r i j}^{2}}}
$$

Where $\mathrm{W}_{\text {oij }}$ is a pixel of the Original Watermark and $\mathrm{W}_{\mathrm{rij}}$ is a pixel of the Recovered Watermark of size MxN.

\section{PROPOSED SCHEME}

The proposed scheme is an entropy based process using DCT and DWT. In the proposed scheme instead of using one fixed subband for watermark embedding, entropy of the subbands is taken as a measure for choosing the appropriate subband for watermark embedding in order to provide both robustness and imperceptibility. The subband number with the highest entropy is used as a key for watermark embedding and extraction. Another key is used for generating the two PN 


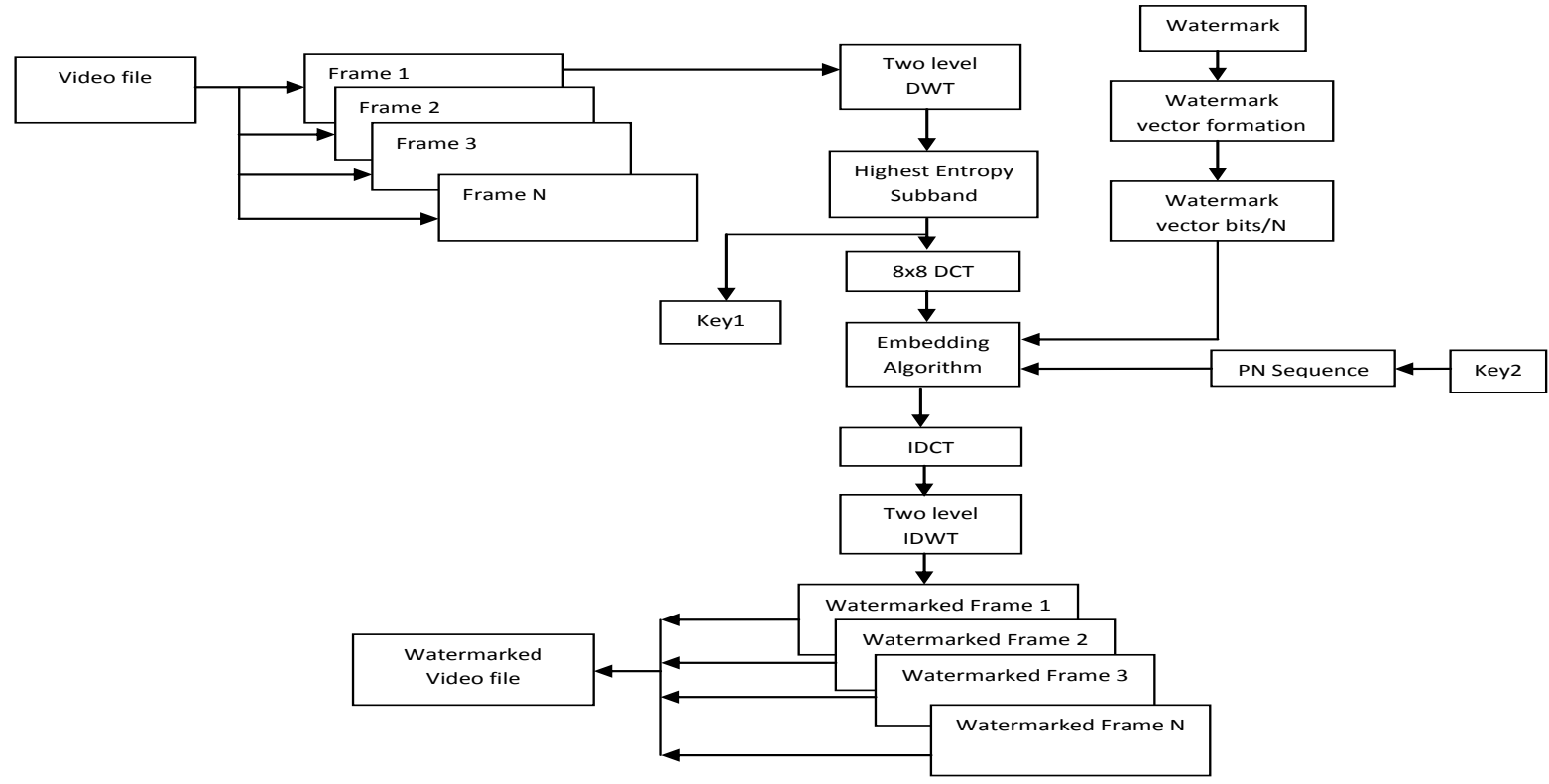

Fig 2: Watermark Embedding Block

sequences for watermark embedding and extraction. Use of two secret keys enhances the security of the scheme. $8 \times 8$ block based DCT is applied on the highest entropy subband. To enhance the robustness of the scheme the middle frequency DCT coefficients are selected for embedding the watermark bits.

The proposed scheme consists of preprocessing of the video file, watermark embedding, transmission and watermark extraction. In the preprocessing part $\mathrm{N}$ frames are extracted from the video file, converted from RGB to gray scale and then resized into $4 \mathrm{Mx} 4 \mathrm{M}$ for the experimental purpose. After preprocessing, watermark is embedded into the frames. The watermark embedding block, transmission block and the watermark extraction block are shown by the depiction in Figure 2, Figure 3 and Figure 4 respectively.

\subsection{Watermark Embedding}

The watermark embedding part can be divided into the following steps:

Step 1: Two level DWT is performed on the first frame to decompose it into sixteen subbands, each of size MxM.

Step 2: Entropy is calculated for all the subbands and the highest entropy subband is chosen for watermark embedding. The highest entropy subband number is stored as the first element of the key1.

Step 3: This subband is then divided into $8 \times 8$ non overlapping blocks, which results in $\mathrm{q}=(\mathrm{MxM}) / 64$ blocks and DCT is carried out on them.
Step 4: The watermark is converted into a message vector of 0 's and 1's. Let, the length of the watermark vector be ' $n$ ' and ' $n$ ' should be less than or equal to $q^{*} N$.

Step 5: The first $\mathrm{n} / \mathrm{N}$ bits of the watermark vector are embedded into the sixteen middle frequency DCT coefficients from 6 to 21 .

Step 6: Two PN sequences pn_zero and pn_one are generated respectively for 0 and 1 with the help of key2. Watermark embedding is done as follows

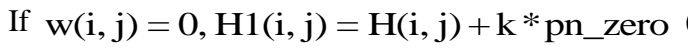

If $\mathrm{w}(\mathrm{i}, \mathrm{j})=1, \mathrm{H} 1(\mathrm{i}, \mathrm{j})=\mathrm{H}(\mathrm{i}, \mathrm{j})+\mathrm{k} * \mathrm{pn} \_$one $(6)$

Where k stands for embedment strength, w $(i, j)$ stands for the value of watermark bit, $\mathrm{H}(\mathrm{i}, \mathrm{j})$ represents middle frequency coefficient before embedding and H1(i, j) stands for the middle frequency coefficient after embedding.

Step 7: Inverse DCT (IDCT) is carried out on each block after its middle frequency coefficients have been modified to embed the watermark bits

Step 8: Inverse DWT (IDWT) is carried out on the highest entropy subband and the other subbands of wavelet decomposition to produce the watermarked frame.

Step 9: Steps 1-8 are repeated for the next N-1 frames to get the watermarked video. 


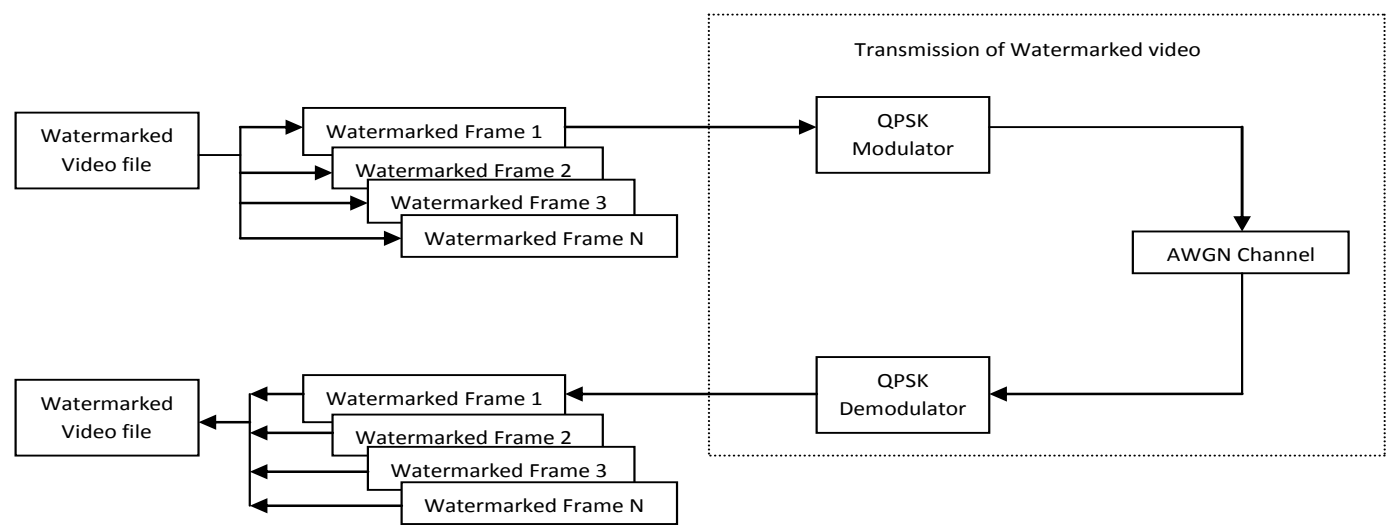

Fig 3: Transmission Block

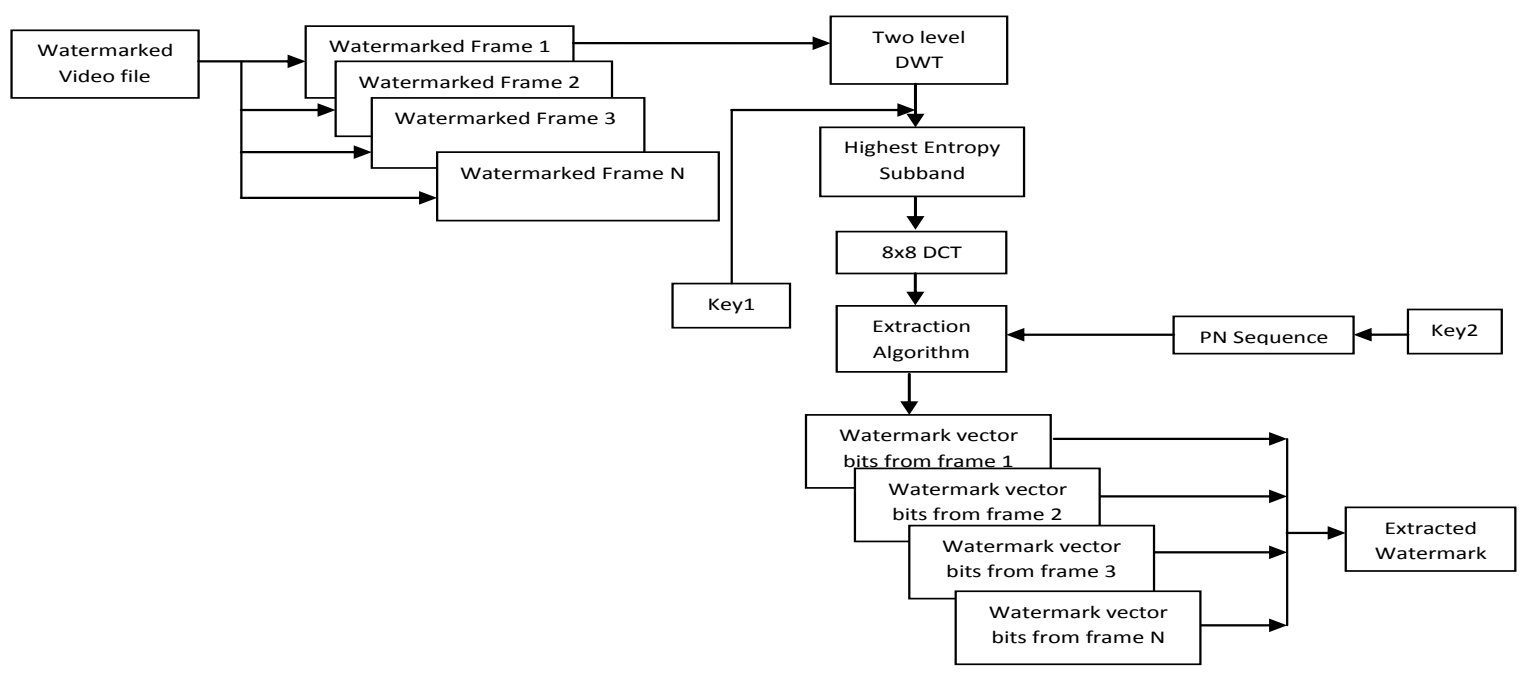

Fig 4: Watermark Extraction Block

\subsection{Transmission of Watermarked Video}

The Watermarked video frames are QPSK modulated and then transmitted through an AWGN channel. At the receiving end QPSK demodulation is performed and the watermark is extracted from the received frames.

\subsection{Watermark Extraction}

The watermark extraction part consists of the following steps:

Step 1: Two level DWT is performed on the first watermarked frame to decompose it into sixteen subbands, each of size MxM.

Step 2: With the help of key1, the highest entropy subband from these sixteen subbands is chosen for watermark extraction.

Step 3: This subband is then divided into $8 \times 8$ blocks, which results in $\mathrm{q}=(\mathrm{MxM}) / 64$ and DCT is carried out on them.
Step 4: The sixteen middle frequency DCT coefficients from 6 to 21 are then selected from each block as done in the embedding algorithm.

Step 5: Two PN sequences r_pn_zero and r_pn_one are generated respectively for 0 and 1 with the help of the key2 used in embedding.

Step 6: Correlation is calculated between the middle frequency coefficients and the two PN sequences. If the correlation with r_pn_one is greater than the correlation with $\mathrm{r}$ _pn_zero, then the extracted watermark bit is considered 1 , otherwise the extracted watermark bit is considered 0 . The first $\mathrm{n} / \mathrm{N}$ watermark bits are extracted.

Step 7: Steps 1-6 are repeated for the next N-1 watermarked frames to extract the rest of the watermark bits.

Step 8: The vector of 0's and 1's is then converted into image form to get the recovered watermark. 

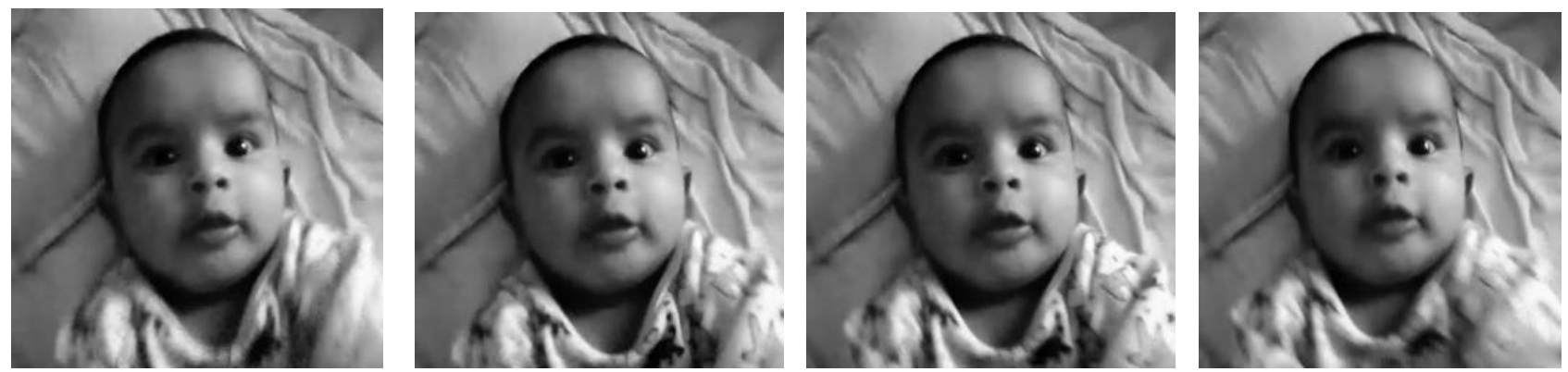

Fig 5: Four consecutive frames of the original video
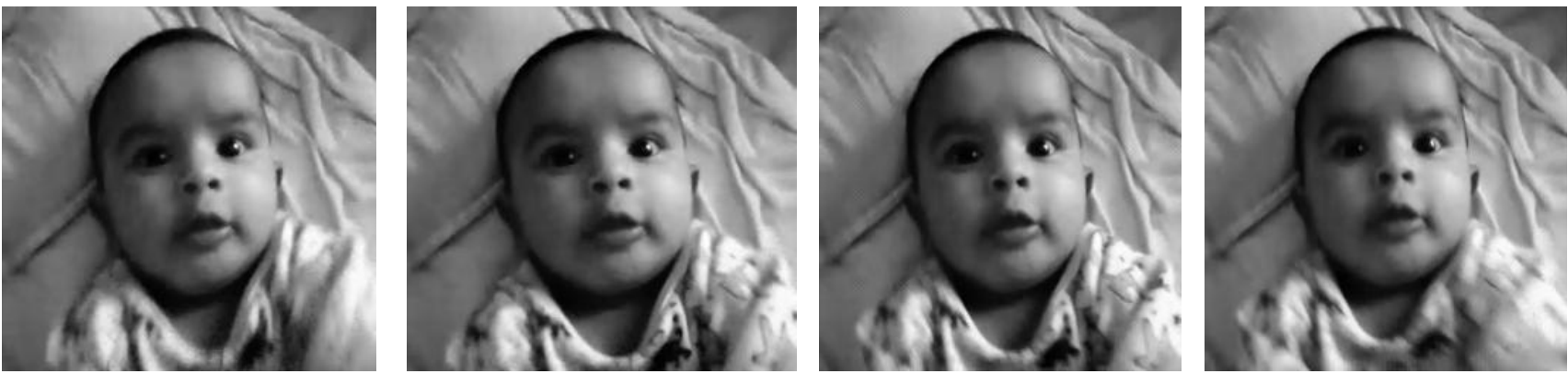

Fig 6: Four consecutive frames of the watermarked video

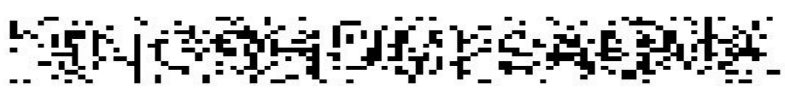

$\mathrm{NC}=0.8827$

\section{ANGSHUMI SARIMA}

$\mathrm{NC}=0.9913$

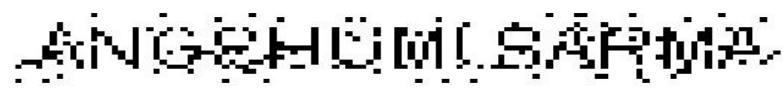

$\mathrm{NC}=0.959$

\section{ANGSHUMI GARMA}

\section{$\mathrm{NC}=1$}

Fig 7: Recovered Watermarks

\section{RESULTS AND DISCUSSION}

For the experimental purpose thirty frames of a video sequence of resolution $256 \times 256$ are considered $(\mathrm{N}=30)$. After two level DWT decomposition the subband size is $64 \times 64$. In this case, the maximum size of the watermark may be $\mathrm{n}=64 * 30$ that is 1920 bits. A binary watermark of size 1680 bits is considered for the experiment. MATLAB is used as the platform for implementing the scheme.

From the experimental results it is observed that as the SNR of the AWGN channel is increased that is the noise intensity is reduced, the quality of the watermarked video frames in the receiving side gradually improves. With an SNR value of 12 $\mathrm{dB}$, the PSNR of the watermarked video frames is $44.5 \mathrm{~dB}$, which proves the imperceptibility of the scheme. Also, with the increase in SNR of the AWGN channel, the quality of the recovered watermark gradually improves. With SNR of 12 $\mathrm{dB}$, the watermark could exactly be recovered with $\mathrm{NC}$ value 1 .

Table 1. Average PSNR and NC values for different SNR values of the AWGN Channel

\begin{tabular}{|c|c|c|}
\hline SNR (dB) & $\begin{array}{c}\text { Average } \\
\text { PSNR (dB) }\end{array}$ & NC \\
\hline 5 & 17.25 & 0.8827 \\
\hline 7 & 21.93 & 0.959 \\
\hline 10 & 33.8 & 0.9913 \\
\hline 12 & 44.5 & 1 \\
\hline
\end{tabular}



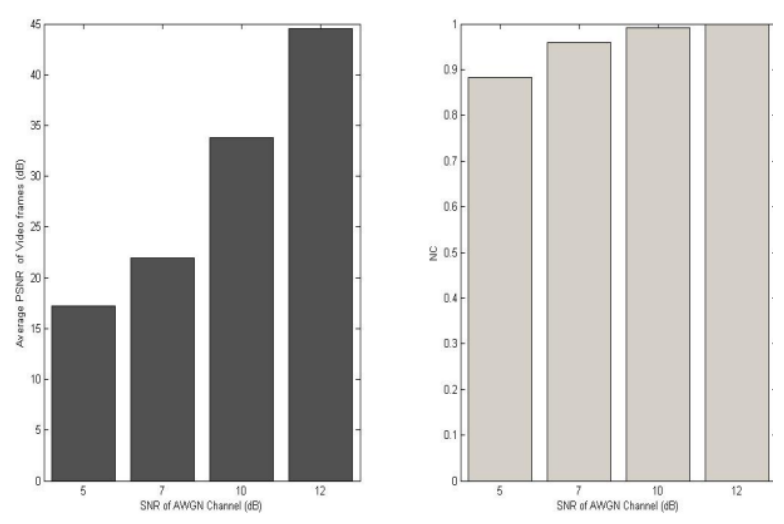

Fig 8: Comparison of Average PSNR and NC for different SNR values of the AWGN Channel

\section{CONCLUSION}

In this paper an entropy based blind video watermarking scheme is proposed and implemented. Use of two secret keys enhances the security of the scheme compared to the other schemes proposed in literature. The high PSNR value obtained proves the imperceptibility of the scheme. Also the scheme is robust against additive Gaussian noise attack, which can be seen from the $\mathrm{NC}$ values. In future this watermarking scheme can be tested for other noise attacks, JPEG compression, rotation, cropping, change in brightness, scaling etc. and other video processing attacks like frame swapping, frame dropping, frame averaging etc.

\section{REFERENCES}

[1] B. L. Gunjal, R.R. Manthalkar: "An Overview of Transform Domain Robust Digital Image Watermarking Algorithms," Journal of Emerging Trends in Computing and Information Sciences, Vol. 2, No. 1.

[2] S. Sinha, P. Bardhan, S. Pramanick, A. Jagatramka, D. K. Kole, A. Chakraborty, "Digital Video Watermarking using Discrete Wavelet Transform and Principal Component Analysis," International Journal of Wisdom Based Computing, Vol. 1 (2), August 2011.

[3] A. M. Kothari and V. V. Dwivedi, "Performance Analysis of Digital Video Watermarking using Discrete Cosine Transform," International Journal of Electrical and Computer Engineering Systems, Vol. 2, No. 1, 2011.

[4] R. T. Paul, "Review of Robust Video Watermarking Techniques," IJCA Special Issue on Computational Science - New Dimensions and Perspectives (3), pp. 9095, 2011.

[5] Y. R. Lin, H. Y. Huang and W. H. Hsu, "An embedded watermark technique in video for copyright protection", IEEE, 18th International Conference on Pattern Recognition, Vol. 00, pp. 795-798, 2006.

[6] A. Al-Haj: "Combined DWT-DCT digital image watermarking," Journal of Computer Science 3 (9): pp. 740-746, 2007.

[7] S.K. Amirgholipour, A. R. Naghsh-Nilchi: "Robust Digital Image Watermarking Based on Joint DWTDCT," International Journal of Digital Content Technology and its Applications Volume 3, Number 2, pp. 42-54, June 2009.

[8] W. H. Bin, Y. H. Liang, W. C. Dong, W. S. Ming: "A New Watermarking Algorithm Based on DCT and DWT Fusion," International Conference on Electrical and Control Engineering, pp. 2614 - 2617, 2010.

[9] M. Chandra, S. Pandey: "A DWT Domain Visible Watermarking Techniques for Digital Images," International Conference on Electronics and Information Engineering, pp. V2-421 - V2-427, 2010.

[10] R. Dubolia, R. Singh, S.S. Bhadoria and R. Gupta: "Digital Image Watermarking by Using Discrete Wavelet Transform and Discrete Cosine Transform and Comparison Based on PSNR," Communication Systems and Network Technologies (CSNT) International Conference on, pp. 593 - 596, 2011.

[11] M. Chandra, S. Pandey and R Chaudhary: "Digital Watermarking Technique for Protecting Digital Images," 3rd IEEE International Conference on Computer Science and Information Technology (ICCSIT), pp. 226 - 233, 2010.

[12] R. V. Franklin, GRS. Manekandan and V. Santhi, "Entropy based Robust Watermarking Scheme using Hadamard Transformation Technique," International Journal of Computer Applications, Vol. 12- No.9, January 2011. 